\title{
COINFECTION OF CUTANEOUS LEISHMANIASIS AND HIV INFECTION
}

\author{
"MARGARET LARTEY, L. ADUSEI, L. HANSON-NORTEY, J.H. ADDY \\ Departments of Medicine and Pathology, University of Ghana Medical School \\ and Korle Bu Teaching Hospital, Accra, Ghana.
}

\begin{abstract}
SUMMARY
Cutaneous leishmaniasis has recently been discovered in some parts of Ghana. The case of an HIV infected patient presenting with cutaneous leishmaniasis at the Korle-Bu Teaching Hospital is discussed. The diagnosis of leishmaniasis was confirmed by histology. Also highlighted is the fact that this is the first reported case of dual infection of HIV and Leishmaniasis in Ghana.
\end{abstract}

The possibility of rapid spread to other members of the community, both immunecompetent and immunesuppressed in view of the large numbers of organisms present in the lesion is discussed.

\section{INTRODUCTION}

Leishmaniasis is a disease caused by parasitic protozoa of the leishmania species and is now endemic in eighty-eight countries in the five continents ${ }^{1}$. It presents in two main forms: visceral and cutaneous. Visceral leismaniasis (VL) is caused by $\mathrm{L}$ donovani and runs a fatal course without treatment while cutaneous leishmaniasis (CL) is caused by $\mathrm{L}$ major (a range of species) and can be very disfiguring. Leishmaniasis is transmitted by sand flies but can be transmitted directly from person to person through the sharing of needles, as is often the case in intravenous drug users ${ }^{2}$. This group is the main group at risk of co-infection.

AIDS and Visceral Leishmaniasis are locked in a vicious cycle of mutual reinforcement since they both attack the cell-mediated immune system. AIDS increases the risk of VL by 100-1000 times in endemic areas ${ }^{2}$. In the revised WHO Clinical Staging for HIV for the African region, visceral leismaniasis is assigned to WHO stage 4 which implies severe immune suppression ${ }^{3}$.

Cutaneous leishmaniasis can become disseminated (diffuse cutaneous leishmaniais) (DCL) especially in immunesuppressed persons. The illness can go on for years and does not heal spontaneously. Patients with HIV/AIDS are particularly susceptible.

In Ghana, an outbreak of cutanoeus leishmanisis hitherto unknown occurred in 2003 starting from the Ho district. This soon spread to 2 adjacent districts in the Volta region ${ }^{4}$. Efforts to identify the secondary host by researchers of the Noguchi Memorial Institute for Medical Research and the Ghana Health Service have so far not yielded fruitful results ${ }^{5}$ whilst the species of the proposed vectors in the outbreak area were so so low that their vectorial role remains uncertain ${ }^{5}$.

\section{CASE}

A case of dual infection is presented after informed consent was obtained from the patient.

A 38 year old HIV positive female patient presented to the Fevers Unit of the Korle Bu Teaching Hospital for further management. She lived in New Longoro-Bamboi in the Brong-Ahafo region, was married and a mother of 6 children.

The main physical finding was pruritic papular dermatitis. Clinically she was staged as World Health Organisation (WHO) stage 2 but further workup showed a CD4 count of 14 and thus patient was prepared for antiretroviral therapy, which she started in October 2004.

In February 2005, three months after initiating antiretroviral therapy, she presented with a large painful infected ulcer at the base of the neck. She presented two more times for drug refills until July of the same year when she presented with a generalised rash and complained of being unwell.

Examination showed generalised concentric hyperpigmented granulomatous tender lesions with raised edges. (Figures 1 and 2). Some of the lesions showed central ulcerations while others had depressed scars.

\footnotetext{
* Author for correspondence
} 
Differential diagnoses of granulomatous candidiasis, tinea nigra or cutaneous tuberculosis were considered.

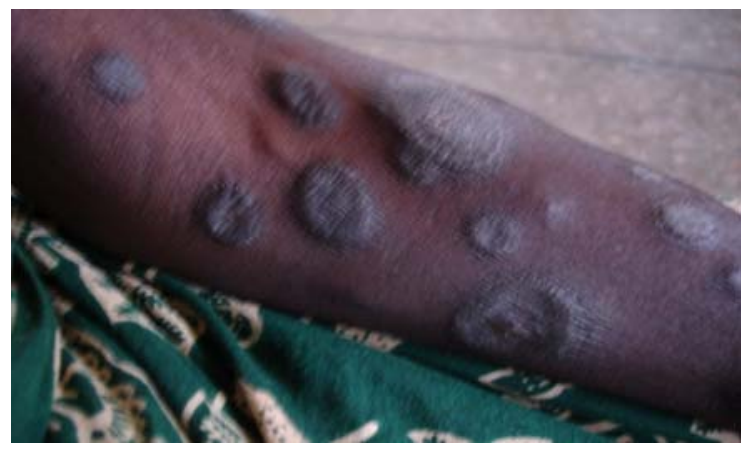

Figure 1 Arm lesions

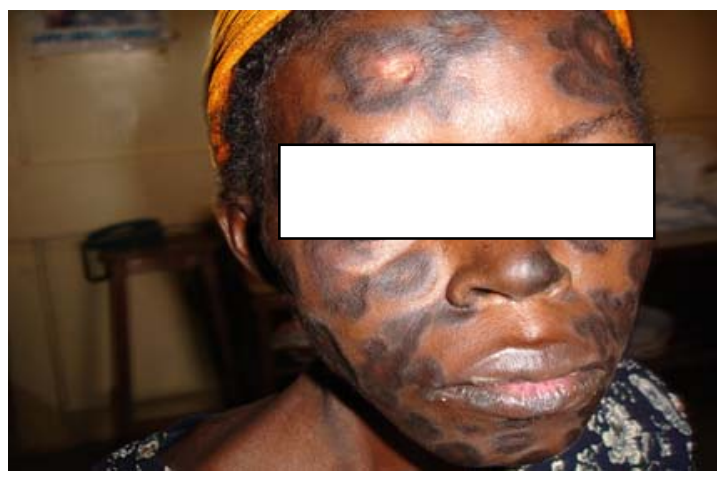

Figure 2 Facial lesions

Punch biopsies of the lesions were sent for histology at the pathology laboratory of the Korle-Bu Teaching Hospital. Histology which showed intradermal and subcutaneous aggregrates of lymphocytes and plasma cells as well as many free small cells, ovoid to round in shape identified as leishmania and confirmed with geimsa staining. Few of the organisms were within histiocytes. (Figure 3) The features are in keeping with cutanoeus leishmaniasis.

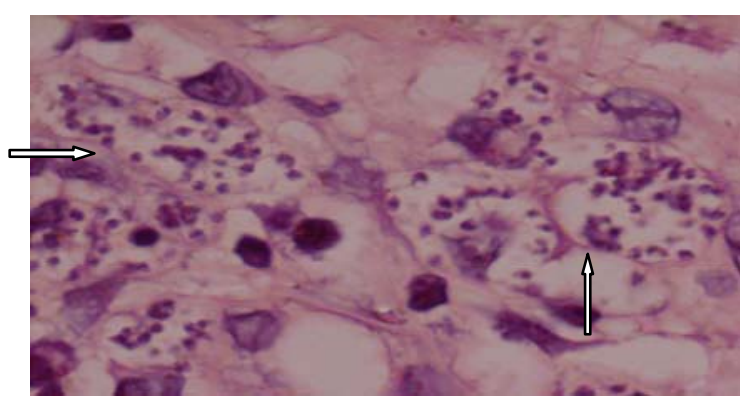

Figure 3 Clusters of free Leishmania in interstitial tissue and subcutaneous fat as shown in Haematoxylin and Eosin stain
Patient however defaulted treatment after10 months of antiretroviral therapy and is lost to follow-up.

\section{DISCUSSION}

Cutaneous leishmaniasis is limited to certain geographical areas, mainly the old world i.e. Sudan, Ethiopia, Kenya, India, Middle East etc and the new world namely Brazil, Mexico and the United States of America but spreading fast because of improved travel and migration ${ }^{2}$. It is spread by sand flies although human to human transmission is possible.

The disease starts as an erythematous papule at the site of the bite, usually an exposed site, however in the black skin it starts as a hyperpigmented papule. The papule then becomes a nodule, which then ulcerates with raised distinct borders. Ulcers are typically large and painless unless there is secondary infection. There may be multiple lesions and lesions up to 200 have been described in a single patient. The skin lesions can be very disfiguring and may heal but recidivans is common. Treatment is with destructive methods and antimony compounds.

Cases of Leishmania/HIV co-infections are being reported more frequently in various parts of the world and it is anticipated that the number of coinfections will rise and be no longer restricted to endemic areas ${ }^{2}$. West Africa has no official surveillance system yet, but several cases have been reported: Cameroon(1), Guinea Bissau(1), Mali (4) and Senegal $(2)^{2}$. This is the first reported case from Ghana.

Hirtheto unknown in Ghana, new cases emerged in the Ho district and soon spread to the Hohoe and Kpandu districts in the Volta region ${ }^{4}$. Presently due to the break-up of two of the districts: Ho district into Ho and Adaklu-Anyigbe and Kpandu into Kpandu and South Dayi, the disease is known to be present in five districts ${ }^{4}$. The case presented hailed from the Brong Ahafo region and may reflect the spread of the disease northwards or from the Northern endemic ${ }^{5}$ belt downwards.

Leishmania/HIV co-infections can lead to epidemiological changes, which modify the traditional patterns of zoonotic VL. Coinfected patients harbour a high number of organisms in their blood thus there is the risk of being reservoirs of the disease thus resulting in increased risks of future epidemics. Sand flies can be infected through a very small quantity of blood less than the content of a 
hypodermic needle ${ }^{2}$. As to whether the same risks of contributions to future epidemics can be said for cutaneous disseminated disease where immunesuppression results in multiple organisms being extracellular is yet to be confirmed. However spread of infections to other immune suppressed patients is real and efforts at case detection, disease control and treatment should be accelerated.

\section{REFERENCES}

1. Dedet JP, Pratlong F. Leishmaniasis. In: Manson P, Cook GC, Zumla A, eds. Manson's Tropical diseases. $21^{\text {st }}$ ed. London: Saunders, 2003; 1339-1364
2. The Leishmaniases and Leishmania/HIV coinfections. WHO fact sheet. Accessed 28/12/05 at 10.35pm @ www.who.int/mediacentrefactsheets/fs116/en/ $\underline{\text { fact } 116}$

3. Interim WHO Clinical Staging of HIV/AIDS and HIV/AIDS Case Definitions for Surveillance. African Region: WHO/HIV/2005.02

4. Annual Reports: Surveillance Unit, Ghana Health Service. 2004

5. Boakye DA, Wilson MD, Kweku M. A Review of Leishmaniasis in West Africa. Ghana Med J 2005; 39 (3): 94-97. 